\title{
Analysis of PPI networks of transcriptomic expression identifies hub genes associated with Newcastle disease virus persistent infection in bladder cancer
}

\author{
Umar Ahmad
}

Universiti Putra Malaysia

Syahril Abdullah

Universiti Putra Malaysia

De Ming Chau

Universiti Putra Malaysia

Suet Lin Chia

Universiti Putra Malaysia

Khatijah Yusoff

Universiti Putra Malaysia

Soon Choy Chan

Perdana University School of Liberal Arts, Science and Technology (PUScLST), Perdana University

Teng Aik Ong

University of Malaya

Azad Hassan Razack

University of Malaya

Abhi Veerakumarasivam ( $\nabla$ abhiv@sunway.edu.my )

Universiti Putra Malaysia

\section{Research Article}

Keywords:

Posted Date: March 3rd, 2022

DOI: https://doi.org/10.21203/rs.3.rs-1377101/v1

License: (1) This work is licensed under a Creative Commons Attribution 4.0 International License.

Read Full License 


\section{Abstract}

Bladder cancer cells acquire persistent infection associated with oncolytic Newcastle disease virus (NDV) in which its molecular events are still unclear. This poses a potential problem for oncolytic virus application for cancer therapy. To unravel the molecular mechanism underlying the development of NDV persistent infection in bladder cancer, we used mRNA expression profile of the persistently infected bladder cancer cells to construct PPI network. Based on path and module exploring in the PPI network, the bridges were found mainly from pathways of p53 signalling, ECM-receptor interaction, and TGF-beta signalling by the upregulated mRNAs, to the antigen processing and presentation, protein processing in endoplasmic reticulum, completement and coagulation cascades by the downregulated mRNAs in NDV persistent TCCSUPPi cells. In persistent EJ28Pi cells comparatively, connections were identified mainly from pathways of renal carcinoma, viral carcinogenesis, Ras signalling and cell cycle by the upregulated mRNAs, to the Wnt signalling, HTLV-I infection and pathways in cancer by the downregulated mRNAs. This connection was mainly dependent on of RPL8- HSPA1A/ HSPA4 in TCCSUPPi cells and EP300, PTPN11, RAC1 - TP53, SP1, CCND1 and XPO1 in EJ28Pi cells. Oncomine validation showed that the top hub genes identified in the network that includes RPL8, THBS1, F2 from TCCSUPPi and TP53 and RAC1 from EJ28Pi are involved in the development and progression of bladder cancer. Protein-drug interaction network, have identified several drugs targets that could be used to disconnect the linkages between modules and prevent bladder cancer cells from acquiring NDV persistent infection. This is the first time reporting the PPI network analysis of differentially expressed mRNAs of the NDV persistently infected bladder cancer cell lines which provide an insight into screening drugs that could be used together with NDV to manage bladder cancer resistance to therapy and progression.

\section{Introduction}

Bladder cancer (BC) is a growth of abnormal tissue term a tumour that develops in the bladder epithelial lining and spread into the muscle layer in some cases[1]. It is also the tenth most common cancer in the world that account for $4.7 \%$ of all the new cancer cases[2]. BC poor prognosis is due to the limited treatment options for advance disease and resistance to conventional therapies. Newcastle disease virus (NDV) is one of the promising novel classes of cancer specific agents that kills human tumour cells while sparing normal cells unharmed[3-5]. This selective killing of cancer cells by NDV is due to the defects in antiviral responses such as the production of interferon which favours the viral replication[6]. The mechanism by which NDV kill cancer cells is via the activation of both intrinsic and extrinsic apoptosis pathways [7] and direct virus-mediated oncolysis[8]. Moreover, NDV triggers long-term adaptive immuneresponse against infected cancer cells [9], making it a strong agent for inducing cell death in cancer. However, NDV has been found to persistently infect subpopulation of cancer cells that resist NDVmediated oncolysis [10]. Similar observations have been reported in other oncolytic viruses such as Reovirus [11] and Measles virus [12]. Persistent infection indicates the ability of a subpopulation of cancer cells to resist NDV-mediated oncolysis. Thus, persistent infection in cancer cells poses a potential problem for maximising the efficacy potential of oncolytic viruses in cancer therapy as tumour contains 
heterogenous subset of cells that harbour a spectrum of genetic aberrations. Although recombinants NDV could have enhanced oncolytic capability, the risk of persistent infection will still remain. The exact mechanism by which cancer cells acquire NDV persistent infection and the molecular basis underlying the development of persistent infection in BC has not been completely elucidated.

Thus, to unravel the regulatory mechanism of oncolytic NDV persistent infection in bladder cancer at molecular level, we previously compared the mRNA expression differences between normal and cancer of the human bladder by transcriptomics [13]. The data indicated that a total of 63 and 134 mRNAs were obtained from TCCSUPPi and EJ28Pi relative to their control respectively, 25 mRNAs were upregulated (log2 fold-change $\geq 0$ ) and 38 mRNAs were downregulated (log2 fold-change $\leq 0$ ) in TCCSUPPi cells. Whereas, 55 mRNAs were upregulated ( $\log _{2}$ fold-change $\geq 0$ ) and 79 mRNAs were downregulated $\left(\log _{2}\right.$ fold-change $\leq 0$ ) in EJ28Pi cells [13]. These differentially expressed genes (DEGs) were significantly enriched in some important upregulated pathways such as TGF-beta signaling, KRAS signaling up and interferon gamma response. Moreover, the study suggested that evasion of apoptosis is crucial in development of persistent infection in bladder cancer cells. The data further revealed that some essential molecular functions such as calcium binding (G0:0005509) and DNA-binding transcription repressor activity, RNA polymerase II-specific (G0:0001227) in TCCSUPPi and protein domain specific binding (GO:0019904) and RNA polymerase II regulatory region sequence-specific DNA binding (G0:0000977) in EJ28Pi were significantly enriched. These may cooperatively contribute to the development of NDV persistent infection in bladder cancer.

Proteins function collectively via protein-protein interactions (PPI) within the cell. This interaction is essential for most of the biochemical activities to achieve specific function in a living cell $[14,15]$ and also provide single protein with multiple functions[16, 17]. Thus, employing PPI methodologies to unravel the molecular mechanisms of biological processes draw an increasing attention in recent time[15, 18, 19]. To deeply understand the regulatory mechanism mechanisms underlying the state of many diseases, PPI networks are generally performed by analysing the DEGs obtained from those diseases [17, 20, 21]. However, there is currently no PPI network analysis for DEGs obtained from an established persistently infected bladder cancer cell. In this study, all the total DEGs obtained in TCCSUPPi (63) and EJ28Pi (134) were analysed separately to construct a PPI network to better understand and unravel the molecular mechanism underlying the development of NDV persistent infection in bladder cancer.

\section{Results}

\section{PPI network of DEGs from TCCSUPPi}

To deeply understand the regulatory mechanisms employed by bladder cancer cell lines in developing NDV persistent infection, differentially expressed genes (DEGs) obtained from both persistent TCCSUPPi and EJ28Pi cell lines were used to construct protein-protein interactions (PPI) network through STRING Interactome database[22]. All the total DEGs obtained in TCCSUPPi (63) and EJ28Pi (134) were analyzed separately for network interactions. As a result, 6 subnetworks that included a continent (subnetwork 1) 
and 5 islands (subnetwork 2 -6) were generated in TCCSUPPi cells. Two subnetworks with highest scores were selected for further analysis. The subnetwork 1 contained 291 nodes, 309 edges and 12 seeds (Figure 1A) and subnetwork 2 had 34 nodes, 36 edges and 2 seeds (Figure 2B). Expressions and the degrees of connection between nodes were represented by their colours and areas respectively. Number of hub nodes in the entire network were further analyzed and the top 14 hub nodes were selected and graphically presented (Figure $3 C$ ). Twelve (12) hub nodes out of 14 were mostly from subnetwork 1 and two superfamily member of cadherin $(\mathrm{CDH} 2$ and $\mathrm{CDH} 5)$ were clustered in subnetwork 2(Figure 2B) with $\mathrm{CDH} 2$ upregulated and $\mathrm{CDH} 5$ downregulated.

\section{Functional connections in the network}

Connections between functions in the identified network were further explored and related nodes were reconstructed (Figure 2). As illustrated, pathways of bladder cancer, malaria, mitophagy, p53 signaling, ECM-receptor interaction, TGF-beta signaling, phagosome, ribosome, focal adhesion and proteoglycans in cancer were significantly enriched $(p<0.05)$ by the upregulated DEGs in the nodes connecting the PPI network (subnetwork 1) (Supplementary Table 1). Antigen processing and presentation, protein processing in endoplasmic reticulum, prion diseases, legionellosis, longevity regulating, complement and coagulation cascades, platelet activation and spliceosome pathways were significantly enriched $(p<0.05)$ by the downregulated DEGs with connected nodes in the PPI network (subnetwork 1) (Supplementary Table 2).

To identify the nodes that are implicated in the aforementioned pathways, medullary analysis was carried out. A total of 9 functional clustered modules and related hub genes were discovered, however, only modules with majority of the nodes were used for redesigning of the modular network. Modules 0 and 1 were observed to contain significant number of the nodes $(p<0.05)$ that contributed to the activation of pathways mentioned above. The top two significant modules were presented in different colours (Figure 2). The results demonstrated that module 0 (coloured blue) and module 1 (coloured red) are key players in in the PPI network of the TCCSUPPi cells, which means that those clustered of genes in module 0 and module 1 act together to promote the development of NDV persistent infection in TCCSUP bladder cancer cell line. The results further illustrated how the upregulated RPL 8 group and downregulated HSPA1A/ HSPA4 are functionally connected (Figure 2).

\section{Protein drug interactions in TCCSUPPi}

Moreover, to identify drug interactions between these connected nodes, we carried out protein-drug interaction analysis using the upregulated nodes that included RPL 8 and THBS1 and downregulated nodes that included $F 2$ and HSPA4. Two subnetworks were identified. Subnetwork 1 comprises of 104 nodes, 103 edges and 1 seed while subnetwork 2 has 4 nodes, 3 edges and 1 seed. Based on the analysis in subnetwork 1, several drugs were identified to be connected to the coagulation factor II, thrombin (F2) node. The top major drugs that are linked to $F 2$ are lepirudin, bivalirudin, drotrecogin alfa, coagulation factor IX (recombinant), menadione, argatroban, and proflavine (Figure 3A). The remaining list of drugs can be found in the appendix. In contrast to subnetwork 2, ribosomal protein L8 (RPL8) that 
was upregulated is linked to alpha-hydroxy-beta-phenyl-propionic acid, anisomycin and puromycin drugs (Figure 3B). These results demonstrate that the identified drugs above can be used to suppress both the upregulated and downregulated nodes, making it possible for the TCCSUP bladder cancer cells to prevent NDV persistent infection.

\section{PPI network of DEGs from EJ28Pi}

Subsequently, the 134 DEGs from EJ28Pi cells were analysed for protein-protein network interactions. The results display a network comprising of 14 subnetworks including one continent (subnetwork 1 ) and 13 islands (subnetwork 2 -14). Once again, the network with the highest scores were selected and analysed in order to provide an insight into the mechanism employed by EJ28 cells in developing NDV persistent infection. Subnetwork 1 had 1161 nodes, 1662 edges and 57 seeds (Figure 4A) while subnetwork 2 identified 21 nodes, 20 edges and 1 seed (Figure 4B). Expression of each node is illustrated by their colours while degrees of connection between nodes were represented by areas. The top 16 hub nodes from the entire network analysis were assessed for their distribution and 15 hub nodes out of this total were from subnetwork 1 while only NADH ubiquinone oxidoreductase core subunit S2 (NDUFS2) was observed from subnetwork 2 (Figure 4C).

Next, functional connections within the constructed network were studied and related nodes were redesigned (Figure 5). As shown, pathways of renal cell carcinoma, viral carcinogenesis, proteoglycans in cancer, prostate cancer, insulin resistance, Ras signalling, circadian rhythm, neurotrophin signalling, cell cycle, aldosterone-regulated sodium reabsorption, FoxO signalling, microRNAs in cancer, Wnt signalling, influenza A, tight junction, viral myocarditis, Kaposi's sarcoma-associated herpesvirus infection, PI3K-Akt signalling, epithelial cell signaling in Helicobacter pylori infection, adipocytokine signalling, epstein-Barr virus infection, adherens junction, bacterial invasion of epithelial cells, cAMP signalling, HTLV-I infection, Longevity regulating pathway, glucagon signaling pathway, leukocyte transendothelial migration, AMPK signaling pathway, pathways in cancer, natural killer cell mediated cytotoxicity and measles were significantly enriched $(p<0.05)$ by the nodes containing upregulated DEGs connecting the PPI network (subnetwork 1). The complete list of these pathways with their false discovery rate (FDR) are listed in Supplementary Table 3.

On the other hand, pathways of Wnt signaling, proteoglycans in cancer, HTLV-I infection, cancer, breast cancer, cellular senescence, melanoma, glioma, MAPK signaling, transcriptional misregulation in cancer, focal adhesion, prostate cancer, endocrine resistance, Th17 cell differentiation, thyroid hormone signaling, thyroid cancer, FoxO signaling, bladder cancer, measles, oxytocin signaling , hippo signaling, amyotrophic lateral sclerosis (ALS), hepatitis C, Jak-STAT signaling, hepatitis B, endometrial cancer, basal cell carcinoma, axon guidance, mitophagy - animal, central carbon metabolism in cancer, Inflammatory bowel disease (IBD), non-small cell lung cancer, Kaposi's sarcoma-associated herpesvirus infection, longterm potentiation, amphetamine addiction, PI3K-Akt signaling , p53 signaling, platinum, drug resistance, pancreatic cancer, chronic myeloid leukemia, regulation of actin cytoskeleton, colorectal cancer, Th1 and Th2 cell differentiation, small cell lung cancer, choline metabolism in cancer, neurotrophin signaling 
pathway, cell cycle, platelet activation and oocyte meiosis were significantly enriched by the downregulated DEGs that have the connected nodes in the PPI network (subnetwork 1). The identified pathways with their false discovery rate (FDR) can be found in Supplementary Table 4.

As for the modules in the network, the PPI network consisted of 41 modules but only modules with majority of the nodes were selected for redesigning of the modular network. Modules 4, 5, 6, 7, and 8 had the most significant number of nodes $(p<0.05)$ that contributed to the activation and enrichment of the above pathways. The modules are coloured red (module 4), blue (module 5 ), green (module 6 ), black (module 7) and brown (module 8 ) respectively (Figure 5). These five modules have significantly $(p<0.05)$ act together to contribute to the development of NDV persistent infection in EJ28 bladder cancer cell line being that they functionally connected via upregulated EP300, IRS1, PTPN11, and RAC1 groups as well as through downregulated TP53, SP1, CCND1 and XPO1 respectively.

To disconnect the linkage between these major modules in the PPI network (subnetwork 1), protein-drug interaction network analysis was performed. The upregulated nodes that included EP300, IRS1, PTPN11, and $R A C 1$, as well as the downregulated nodes comprising of TP53, SP1, CCND1 and XPO1 were mapped to DrugBank database for matching nodes to obtain their drug interaction information. Two subnetworks were discovered with subnetwork 1 containing 4 nodes, 3 edges and 1 seed and subnetwork 2 containing 3 nodes, 2 edges and 1 seed. Dextromethorphan and guanosine-5'-diphosphate drugs were identified to effectively interact with upregulated rac family small GTPase 1 (RAC1) node (Figure 6A). Contrary to subnetwork 2, tumour protein P53 (TP53) node that was downregulated is connected to acetylsalicylic acid, AZD, and 1-(9-ethyl-9H-carbazol-3-yl)-N-methylmethanamine drugs (Figure 6B), which basically means that these drugs can be used together with NDV to enhance the oncolytic activity of NDV against EJ28 bladder cancer cell lines and prevent the cells from acquiring persistent infection.

\section{Validation of hub genes in Oncomine}

To validate the expression profiles of the top hub genes that were identified in the protein-protein interaction network, mRNA expression mining of these hub genes in publicly available Oncomine database (www.oncomine.org) [23] that include the upregulated nodes RPL8 and THBS1 as well as the downregulated nodes $F 2$ in TCCSUPPi cells was carried out. Likewise, the upregulated node RAC1 and downregulated node TP53 obtained from EJ28Pi cells were subsequently investigated. The results showed that among the three hub genes (RPL8, THBS1 and F2) obtained from TCCSUPPi cells, RPL8 was significantly upregulated in bladder cancer cells as compared to normal bladder tissue (GSE3167; Figure 7 upper left; $p=6.36 \mathrm{E}-5$ ) [24]. In a study undertaken by Kim, Kim [25], relative expression of THBS1 and F2 were slightly higher in bladder cancer than in the normal bladder tissue but the difference was not statistically significant (GSE13507; Figure 7 upper middle \& upper right; $p>0.05$ ), suggesting that $R P L 8$, $T H B S 1$ and $F 2$ genes play a vital role in bladder cancer progression and metastasis. These genes may also be the reason why cancer cells are able to maintain normal growth and development even after NDV infection as seen in the case of the established persistent TCCSUPPi cells. 
On the other hand, TP53 and RAC1 mRNA expression levels in bladder cancer is shown to be lower than in the normal bladder tissue (GSE13507; Figure 7 lower left \& lower right; $p>0.05$ ). These data suggest that TP53 and RAC1 were downregulated in bladder cancer and may likely contribute to the development of NDV persistent infection in EJ28 cells noting that the TP53 was found to be upregulated in the established persistent EJ28Pi cells.

\section{Discussion}

Analysis of the RNA-Seq data revealed several essential pathways that were enriched by the DEGs identified to be involved in NDV persistent infection in bladder cancer cell lines. We then performed protein-protein interaction (PPI) analysis of the identified DEGs to unravel the connections between these pathways and the genes. It can be observed that there is functional connectivity in the biological processes. By modular analysis of the PPI network in persistent TCCSUPPi cells, RPL8 node group was upregulated and implicated in the pathways of bladder cancer, malaria, mitophagy, p53 signaling, ECMreceptor interaction, TGF-beta signaling, phagosome, ribosome, focal adhesion and proteoglycans in cancer were clustered in the same module, suggesting that these genes co-function together to develop NDV persistent infection in TCCSUP bladder cancer cell line. RPL 8 is a protein coding gene being referred to as ribosomal protein L8 that is known to be associated with viral mRNA translation and rRNA processing pathways in both the nucleus and the cytosol[26]. In our study, RPL 8 gene was upregulated in the persistent TCCSUPPi cells. Dysregulation or misexpression of this gene family is associated with different types of cancer cells [27-30] and several other inherited genetic diseases[31]. This shows that $R P L 8$ is associated with viral infection and its role in establishing NDV persistent infection in TCCSUP bladder cancer cell line is obvious in this study.

On the other hand, the upregulated nodes found in persistent EJ28Pi cells includes EP300, PTPN11, and $R A C 1$ implicated in the pathways of renal cell carcinoma, viral carcinogenesis, proteoglycans in cancer, prostate cancer, insulin resistance, Ras signalling, circadian rhythm, neurotrophin signalling, cell cycle, aldosterone-regulated sodium reabsorption, FoxO signalling, microRNAs in cancer, Wnt signalling, influenza A, tight junction, viral myocarditis, Kaposi's sarcoma-associated herpesvirus infection, PI3K-Akt signalling, epithelial cell signalling in helicobacter pylori infection, adipocytokine signalling, epstein-Barr virus infection, adherens junction, bacterial invasion of epithelial cells, cAMP signalling, HTLV-I infection, longevity regulating pathway, glucagon signalling pathway, leukocyte transendothelial migration, AMPK signalling pathway, pathways in cancer, natural killer cell mediated cytotoxicity and measles, were clustered in module 5, 6, and 7 (Fig. 4.48), indicating that the genes in these cluster act together to facilitate the development of NDV persistent infection in EJ28 bladder cancer cell line. Our findings demonstrated that EP300, PTPN11, and RAC1 genes were upregulated in NDV in persistent EJ28Pi cells. EP300 gene is known to encode for adenovirus E1A-associated cellular p300 transcriptional co-activator protein that functions in regulations of transcription by remodeling chromatin[32-34]. It plays a vital role in cell proliferation, differentiation and epithelial cancer [35] as well as co-activate hypoxia-inducible factor 1 alpha, HIF1A to facilitate the expression of VEGF, a key player in inducing cellular hypoxia[36]. Recent study suggested that HAT domain mutation on EP300 gene has a major impact on malignant 
progression and growth[37], suggesting that EP300 gene may contribute to the survival and growth of EJ28 cells after being persistently infected with oncolytic NDV.

PTPN11 also known as protein tyrosine phosphatase, none-receptor type 11 is an oncogene that has the potential to turn normal cells into cancerous cells when mutated. It encodes protein that is in the protein tyrosine phosphatase (PTP) family and provides key important functions in cell growth and differentiation. PTPN11 gene is mutated in about $1.19 \%$ of all types of cancers including melanoma, carcinoma of the lung, malignant glioma and leukaemia[38]. This is consistent with our report, demonstrating that PTPN11 gene is upregulated in NDV persistent EJ28Pi cells. In addition, RAC1 RAS superfamily was also found to be upregulated in EJ28Pi cells. It functions by controlling cytoskeletal reorganization and cell growth. In line with our results, evidence has shown that the upregulation of RAC1 is associated with lymphovascular invasion and lymph node metastasis of the urinary tract cancer[39]. Therefore, upregulation of EP300, PTPN11, and RAC1 genes may play a role in the development of NDV persistent infection in EJ28 bladder cancer cell line via association with the above implicated pathways.

Protein-drug interaction network revealed drugs that may be used to disconnect the linkages between the major modules in the constructed PPI network for both the TCCSUPPi and EJ28Pi cells. We observed several drugs that are linked to the downregulated node F2 in TCCSUPPi cells that includes lepirudin, bivalirudin, drotrecogin alfa, coagulation factor IX (recombinant), menadione, argatroban and proflavine etc. On the other hand, puromycin, anisomycin and alpha-hydroxyl-beta-phenyl-propionic acid were the drug targets for upregulated RPL8 node group. One important drug among the connected drugs on $F 2$ node network is menadione, a biologically active vitamin $\mathrm{K} 3$ that promotes coagulation of blood and is reported to strongly inhibit cancerous growth especially the neoplastic cells through conversion to vitamin $\mathrm{K} 2$, and reduced mutagenicity in rapidly growing cells in a new born[40]. Other studies demonstrated synergistic effects of vitamin $\mathrm{C}$ and menadione (vitamin K3) alone with radiation therapy on killing of bladder cancer cells as well as other cancer types without adverse reactions for patients[4143]. Anisomycin drug is an antibiotic that inhibits synthesis of protein via the activity of peptidyl transferase in ribosomes[44], it interacts with RPL8 node in the TCCSUPPi PPI network. Studies have found that this drug kills a variety of cancer cells such as adenocarcinoma, colon and leukaemia through induction of apoptosis[45, 46]. Thus, anisomycin that interacts with RPL8 as well as menadione and the remaining drugs that interacts with $F 2$ are drugs that can potentially reverse the mechanism employed by TCCSUP cells in acquiring NDV persistent infection thus may be used in combination with NDV to synergistically kill bladder cancer cells that initially resist NDV-mediated oncolysis.

The identified drugs that interact with upregulated rac family small GTPase 1 (RAC1) node in persistent EJ28Pi cells network include dextromethorphan and guanosine-5'-diphosphate. On the other hand, the drugs acetylsalicylic acid, AZD, and 1-(9-ethyl-9H-carbazol-3-yl)-N-methylmethanamine were found to interact with downregulated TP53 node. Dextromethorphan belongs to a class of drugs known as morphinan that has sedative and stimulant properties, and is often used as cough suppressant. Report has it that dextromethorphan is capable of causing mild motor and cognitive impairment, paranoia and delusion[47]. It is unclear how dextromethorphan exerts its effects on bladder cancer cell lines. Thus, 
investigating the role of dextromethorphan on bladder cancer cell line is needed to understand its mechanism of action on cancer. We also found that guanosine-5'-diphosphate drug interacts with RAC1 network. It has been reported that guanosine-5'-diphosphate in combination with other drug agents has significantly reduced the aggressive nature of breast cancers[48]. Another important drug identified to interact with TP53 node in the network is AZD. This drug may potentially be used for bladder cancer treatment because AZD is already being used in clinical trials, testing against many different types of cancers including esophageal cancer [49], small cell lung cancer[50] and colorectal cancer[51]. Therefore, the drugs mentioned above may be used in combination with NDV to destroy the EJ28 bladder cancer cells and prevent them from further acquiring NDV persistent infection.

Our results further discovered that some upstream genes such as $R P L 8$ and $F 2$ were significantly upregulated in bladder cancer with the exception of THBS1, TP53 and RAC1 that were found to be less expressed according to the Oncomine-based expression analysis. Through PPI network analysis, we identified $R P L 8$ and $F 2$ as therapeutic drug targets which were obviously overexpressed in bladder cancer. The expression patterns indicate that $R P L 8, F 2, T H B S 1, T P 53$ and $R A C 1$ are differentially expressed in bladder cancer versus the normal bladder tissues and that the level of their expression varies depending on the nature of tissue.

\section{Conclusion}

Our results for the first time analyzed and profiled the transcriptome of NDV persistent bladder cancer cell lines to construct protein-protein interaction (PPI) network. We observed that the upregulated RPL8 group and downregulated HSPA1A/HSPA4 group are functionally connected with one another based on the data from TCCSUPPi cells. Through RPL8 - HSPA1A/HSPA4 link, a considerable number of pathways were identified that includes bladder cancer, malaria, p53 signaling, ECM-receptor interaction and TGF-beta signaling were implicated which are indicative of the molecular pathways associated with NDV persistent infection in TCCSUP bladder cancer cell line. Similarly, we reported the functional connectivity between upregulated EP300, IRS1, PTPN11, and RAC1 group and the downregulated TP53, SP1, CCND1 and XPO1 group based on the data from EJ28Pi cells. Through IRS1, PTPN11, and RAC1 as well as TP53, SP1, CCND1 and XPO1 connections, several major pathways including renal cell carcinoma, viral carcinogenesis, cell cycle, FoxO signaling, pathway in cancer, NK cells mediated cytotoxicity, Ras signaling etc were implicated. This is an indication of the molecular pathways associated with NDV persistent infection in EJ28 bladder cancer cell line.

Furthermore, our data provide clues into identifying new drug targets that can synergistically act together with NDV to combat and destroy bladder cancer cells as well as reverse the ability to acquire NDV persistent infection. The results in this study therefore enhances our understanding of host response to NDV persistent infection by providing candidate genes and pathways that may be associated with persistent infection in bladder cancer cells. In addition, several proteins that are crucial to the development of persistent infection were identified through PPI network. 
Finally, we recommend that the potential drugs to target the key nodes on the PPI network should be screened as this will help to identify new approaches for cancer treatment in combination with the oncolytic Newcastle disease virus. Overall, this work provides the molecular basis for further research to unravel the function of these novel pathways, candidate genes and specific proteins that seems to activate the phenotypes associated with NDV persistent infection in bladder cancer cells.

\section{Materials And Methods}

\section{DEGs from persistently infected cells}

The DEGs in this study were previously published in a preprint [13] and deposited in NCBI Gene Expression Omnibus (GEO) under accession number GSE140902 and released to the public (https://www.ncbi.nlm.nih.gov/geo/query/acc.cgi?acc=GSE140902). A total of 63 and 134 differentially expressed genes (DEGs) from persistent TCCSUPPi and EJ28Pi cells relative to their uninfected controls were obtained from the total RNA samples of these NDV persistently infected cells. Of 63 DEGs in TCCSUPPi cells, 25 genes were upregulated and 38 genes were downregulated. Whilst in EJ28Pi, 55 genes were upregulated and 79 genes were downregulated. These DEGs were enriched into pathways as reported in the paper and were used for the PPI network construct and analysis.

\section{PPI network analysis}

DEGs obtained from the persistently infected cells were used to construct the PPI network using a multifunctional online software Network Analyst (https://www.networkanalyst.ca) [52, 53]. Path and module analyses as well as determination of the protein-drug interactions were carried using this software. PPI networks were generated for each of the persistently infected cell line by submitting the number of DEGs together with their Ensembl gene IDs and $\log _{2}$ (fold change) expression to the NetworkAnalyst software. Search Tool for the Retrieval of Interacting Genes/Proteins (STRING) Interactome was used as the PPI database and the cut off confidence scores was set at 900 since the range of the scores are between 400 to 1000 [22]. The seeds were mapped to the corresponding molecular interaction database and the subnetworks with more cluster of nodes were chosen and demonstrated as top nodes in the network.

\section{Nodal path exploration}

Nodes that are functionally connected and linked in the generated PPI network was visualized using the path explorer function segment of the NetworkAnalyst software[52]. Paths of interest and the connections between nodes were selected and redesigned to enable further understanding of the cascades of event in the PPI network. The implicated nodes that are significantly enriched in the pathways associated with development of persistent infection were specifically marked in the connections.

Exploring module in the PPI network 
Using the same NetworkAnalyst software[52], a module explorer section of the application was used to identify the clustered of subnetworks that collectively function together in the PPI network through Walktrap Algorithm that uses walk based strategy to detect module. The degrees of internal (edges within a module) and external (edges connecting the nodes of a module with the rest of the graph) edges for determining the significance of the module was based on the Wilcoxon rank-sum and values of $p<0.05$ were considered significant. Then, the modules of interest were marked with different colours in the PPI network.

\section{Protein drug interaction}

The upregulated and downregulated nodes of interest obtained from persistently infected TCCSUPPi (upregulated RPL8 and downregulated HSPA1A/ HSPA4 groups) and EJ28Pi (upregulated EP300, IRS1, PTPN11, and RAC1 and downregulated TP53, SP1, CCND1 and XPO1) cells were further analyzed to identify their interactions with drugs. DrugBank database version 5.0 was used to match the these nodes against the panel of drug target to generate and collect the protein-drug interactions network information[54].

\section{Validation of hub genes}

The candidate hub genes identified from hub module in the network were validated by an Oncomine database (http://www.oncomine.org/)[23]. Expression level of these hub genes in both the persistently infected cell lines were validated using Dyrskjøt, Kruhøffer [24] (GSE3167) and Kim, Kim [25] (GSE13507) gene expression data with defined thresholds values for fold change $>2$, gene rank top $10 \%$ and $p<0.001$. Data type was restricted to only mRNA and statistical significance was based on Student's $t$ test.

\section{Declarations}

\section{Data availability}

The authors declare that all the data in this manuscript are available. Raw and processed data were deposited in NCBI Gene Expression Omnibus (GEO) under accession number GSE140902 (https://www.ncbi.nlm.nih.gov/geo/query/acc.cgi?acc=GSE140902).

\section{Acknowledgement}

This study was supported by the Ministry Energy, Science, Technology, Environment and Climate Change (MESTECC) Malaysia Flagship Fund, reference number: FP0514B0021-2(DSTIN) and University of Malaysia High Impact Research (HIR) Grant Scheme: UM.C/625/1/HIR/130.

\section{Author Contributions}

U.A, A.V., S.C.C., D.M.C., S.L.C., S.A., T.A.O., A.Z.R and K.Y., designed the study. U.A. performed the wet and dry lab work. U.A., A.V., S.C.C., D.M.C., S.L.C., S.A., and K.Y analysed the data. U.A drafted the manuscript. 
All authors reviewed and edited the manuscript.

\section{Competing Interests}

The authors declare no competing interests.

\section{References}

1. Kaseb, H. and N.R. Aeddula, Cancer, bladder, in StatPearls [Internet]. 2019, StatPearls Publishing.

2. Ferlay, J., et al., Global cancer observatory: cancer today. Lyon, France: International Agency for Research on Cancer, 2018.

3. Aghi, M. and R.L. Martuza, Oncolytic viral therapies-the clinical experience. Oncogene, 2005. 24(52): p. $7802-7816$.

4. Schirrmacher, V. and P. Fournier, Newcastle disease virus: a promising vector for viral therapy, immune therapy, and gene therapy of cancer, in Gene Therapy of Cancer. 2009, Springer. p. 565-605.

5. Reichard, K.W., et al., Newcastle disease virus selectively kills human tumor cells. J Surg Res, 1992. 52(5): p. 448-53.

6. Ginting, T.E., et al., Antiviral interferons induced by Newcastle disease virus (NDV) drive a tumorselective apoptosis. Scientific reports, 2019. 9(1): p. 15160-15160.

7. Elankumaran, S., D. Rockemann, and S.K. Samal, Newcastle disease virus exerts oncolysis by both intrinsic and extrinsic caspase-dependent pathways of cell death. Journal of virology, 2006. 80(15): p. 7522-34.

8. Zamarin, D. and P. Palese, Oncolytic Newcastle disease virus for cancer therapy: old challenges and new directions. Future Microbiol, 2012. 7(3): p. 347-67.

9. Schwaiger, T., et al., Newcastle disease virus mediates pancreatic tumor rejection via NK cell activation and prevents cancer relapse by prompting adaptive immunity. International journal of cancer, 2017. 141(12): p. 2505-2516.

10. Chia, S.-L., K. Yusoff, and N. Shafee, Viral persistence in colorectal cancer cells infected by Newcastle disease virus. Virology journal, 2014. 11(1).

11. Alain, T., et al., The oncolytic effect in vivo of reovirus on tumour cells that have survived reovirus cell killing in vitro. Br J Cancer, 2006. 95(8): p. 1020-7.

12. Goldman, M.B., et al., Measles virus persistence in an immortalized murine macrophage cell line. Virology, 1995. 207(1): p. 12-22.

13. Ahmad, U., et al., Functional Transcriptome Analysis of Bladder Cancer Cell Lines Persistently Infected with Oncolytic Newcastle Disease Virus. bioRxiv, 2020: p. 2020.12.14.422610.

14. Wang, L., et al., An ensemble approach for large-scale identification of protein-protein interactions using the alignments of multiple sequences. Oncotarget, 2017. 8(3): p. 5149. 
15. Huang, Y.-A., et al., Sequence-based prediction of protein-protein interactions using weighted sparse representation model combined with global encoding. BMC bioinformatics, 2016. 17(1): p. 184.

16. Sharan, R., I. Ulitsky, and R. Shamir, Network-based prediction of protein function. Molecular systems biology, 2007. 3(1): p. 88.

17. Wu, B., et al., PPI network analysis of mRNA expression profile of ezrin knockdown in esophageal squamous cell carcinoma. BioMed research international, 2014. 2014.

18. Li, Z.-W., et al., Highly accurate prediction of protein-protein interactions via incorporating evolutionary information and physicochemical characteristics. International journal of molecular sciences, 2016. 17(9): p. 1396.

19. An, J.-Y., et al., Identification of self-interacting proteins by exploring evolutionary information embedded in PSI-BLAST-constructed position specific scoring matrix. Oncotarget, 2016. 7(50): p. 82440.

20. Nguyen, T.-P., et al., Network analysis of neurodegenerative disease highlights a role of Toll-like receptor signaling. BioMed research international, 2014. 2014.

21. Kotni, M.K., M. Zhao, and D.-Q. Wei, Gene expression profiles and protein-protein interaction networks in amyotrophic lateral sclerosis patients with C9orf72 mutation. Orphanet journal of rare diseases, 2016. 11(1): p. 148.

22. Szklarczyk, D., et al., STRING v10: protein-protein interaction networks, integrated over the tree of life. Nucleic Acids Res, 2015. 43(Database issue): p. D447-52.

23. Rhodes, D.R., et al., ONCOMINE: a cancer microarray database and integrated data-mining platform. Neoplasia, 2004. 6(1): p. 1-6.

24. Dyrskjøt, L., et al., Gene expression in the urinary bladder: a common carcinoma in situ gene expression signature exists disregarding histopathological classification. Cancer research, 2004. 64(11): p. 4040-4048.

25. Kim, W.-J., et al., Predictive value of progression-related gene classifier in primary non-muscle invasive bladder cancer. Molecular cancer, 2010. 9(1): p. 3.

26. Hanes, J., et al., Characterization by cDNA cloning of the MRNA of human ribosomal protein $L 8$. Biochemical and biophysical research communications, 1993. 197(3): p. 1223-1228.

27. Henry, J.L., D.L. Coggin, and C.R. King, High-level expression of the ribosomal protein L 19 in human breast tumors that overexpress erbB-2. Cancer research, 1993. 53(6): p. 1403-1408.

28. Vaarala, M.H., et al., Several genes encoding ribosomal proteins are over-expressed in prostatecancer cell lines: Confirmation of L7a and L37 over-expression in prostate-cancer tissue samples. International journal of cancer, 1998. 78(1): p. 27-32.

29. Cheng, Q., et al., Identification of molecular markers for the early detection of human squamous cell carcinoma of the uterine cervix. British journal of cancer, 2002. 86(2): p. 274.

30. Lopez, C.D., G. Martinovsky, and L. Naumovski, Inhibition of cell death by ribosomal protein L35a. Cancer letters, 2002. 180(2): p. 195-202. 
31. Yang, F. and W. Liu, The progress of ribosomal protein genes and human diseases. J Clin Exp Pathol, 2005. 20: p. 354-356.

32. M Valor, L., et al., Lysine acety/transferases CBP and p300 as therapeutic targets in cognitive and neurodegenerative disorders. Current pharmaceutical design, 2013. 19(28): p. 5051-5064.

33. Eckner, R., et al., Molecular cloning and functional analysis of the adenovirus E1A-associated 300-kD protein ( $p 300$ ) reveals a protein with properties of a transcriptional adaptor. Genes \& development, 1994. 8(8): p. 869-884.

34. Iyer, G., et al., Prevalence and co-occurrence of actionable genomic alterations in high-grade bladder cancer. Journal of Clinical Oncology, 2013. 31(25): p. 3133.

35. Wang, F., C.B. Marshall, and M. Ikura, Transcriptional/epigenetic regulator CBP/p300 in tumorigenesis: structural and functional versatility in target recognition. Cellular and Molecular Life Sciences, 2013. 70(21): p. 3989-4008.

36. Ramakrishnan, S., V. Anand, and S. Roy, Vascular endothelial growth factor signaling in hypoxia and inflammation. Journal of Neuroimmune Pharmacology, 2014. 9(2): p. 142-160.

37. Duex, J.E., et al., Functional impact of chromatin remodeling gene mutations and predictive signature for therapeutic response in bladder cancer. Molecular Cancer Research, 2018. 16(1): p. 69-77.

38. Consortium, A.P.G., AACR Project GENIE: powering precision medicine through an international consortium. Cancer discovery, 2017. 7(8): p. 818-831.

39. Kamai, T., et al., Increased Rac1 activity and Pak1 overexpression are associated with lymphovascular invasion and lymph node metastasis of upper urinary tract cancer. BMC Cancer, 2010. 10: p. 164.

40. KLACK, K., J.F. CARVALHO, and K. Vitamina, Metabolismo, fontes e interação com o anticoagulante varfarina. Revista Brasileira de reumatologia, 2006. 46(6): p. 398-406.

41. Gilloteaux, J., et al., Cell damage and death by autoschizis in human bladder (RT4) carcinoma cells resulting from treatment with ascorbate and menadione. Ultrastructural pathology, 2010. 34(3): p. 140-160.

42. McGuire, K., et al., Synergistic antitumor activity of vitamins $C$ and $K 3$ on human bladder cancer cell lines. Journal of Cancer Therapy, 2013. 4(06): p. 7.

43. Ren, X., et al., The combination of ascorbate and menadione causes cancer cell death by oxidative stress and replicative stress. Free Radical Biology and Medicine, 2019. 134: p. 350-358.

44. Gold, P.E., Protein synthesis inhibition and memory: formation vs amnesia. Neurobiology of learning and memory, 2008. 89(3): p. 201-211.

45. Lunghi, P., et al., Expression and activation of SHC/MAP kinase pathway in primary acute myeloid leukemia blasts. The Hematology Journal, 2001. 2(2): p. 70-80.

46. Yang, H., et al., Macrophage inhibitory cytokine-1 (MIC-1) and subsequent urokinase-type plasminogen activator mediate cell death responses by ribotoxic anisomycin in HCT-116 colon cancer cells. Biochemical pharmacology, 2009. 78(9): p. 1205-1213. 
47. Martinak, B., et al., Dextromethorphan in cough syrup: The poor man's psychosis.

Psychopharmacology bulletin, 2017. 47(4): p. 59.

48. Majumder, M., et al., EP4 as a therapeutic target for aggressive human breast cancer. International journal of molecular sciences, 2018. 19(4): p. 1019.

49. Yang, L., et al., Wee-1 kinase inhibitor AZD-1775 radiosensitizes esophageal cancer through targeting G2 checkpoint activation. 2018, AACR.

50. Ramalingam, S.S., et al., Phase II study of Cediranib (AZD 2171), an inhibitor of the vascular endothelial growth factor receptor, for second-line therapy of small cell lung cancer (National Cancer Institute\# 7097). Journal of thoracic oncology, 2010. 5(8): p. 1279-1284.

51. Leichman, L., et al., Phase II study of olaparib (AZD-2281) after standard systemic therapies for disseminated colorectal cancer. The oncologist, 2016. 21(2): p. 172-177.

52. Xia, J., E.E. Gill, and R.E. Hancock, NetworkAnalyst for statistical, visual and network-based metaanalysis of gene expression data. Nat Protoc, 2015. 10(6): p. 823-44.

53. Xia, J., M.J. Benner, and R.E. Hancock, NetworkAnalyst-integrative approaches for protein-protein interaction network analysis and visual exploration. Nucleic Acids Res, 2014. 42(Web Server issue): p. W167-74.

54. Wishart, D.S., et al., DrugBank 5.0: a major update to the DrugBank database for 2018. Nucleic Acids Research, 2017. 46(D1): p. D1074-D1082.

\section{Supplementary}

The Appendix is not available with this version.

\section{Figures}




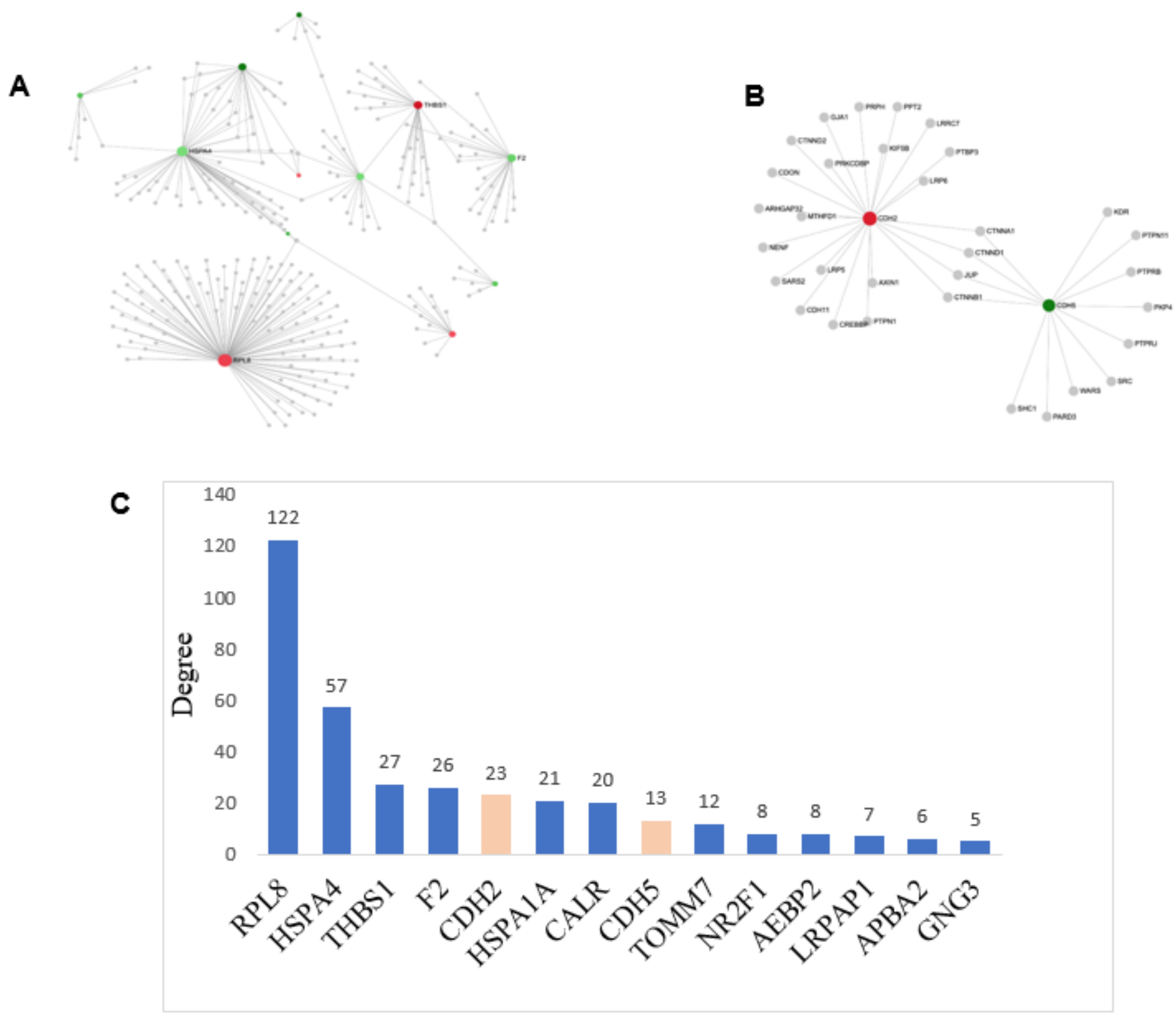

Figure 1

First and second identified networks in TCCSUPPi cells. A) First identified network in TCCSUPPi cells. Red and green colours represent the nodes expression that are up- and down-regulated, respectively. The expression level is represented by the shades of colour and the area of the nodes indicate the degrees that the nodes connect to others. Nodes with gene names are the top 4 nodes in the PPI network. B) Second identified network in TCCSUPPi cells. Subnetwork 2 contains both up- and downregulated nodes that are affected in the pathways. Nodes in red and green colours are upregulated and downregulated in TCCSUPPi cells, respectively. C) Hub nodes in the PPI network in TCCSUPPi cells. Top 14 hub nodes with their degree level are shown. Genes in blue colour are from subnetwork 1 and red accent are subnetwork 2. 


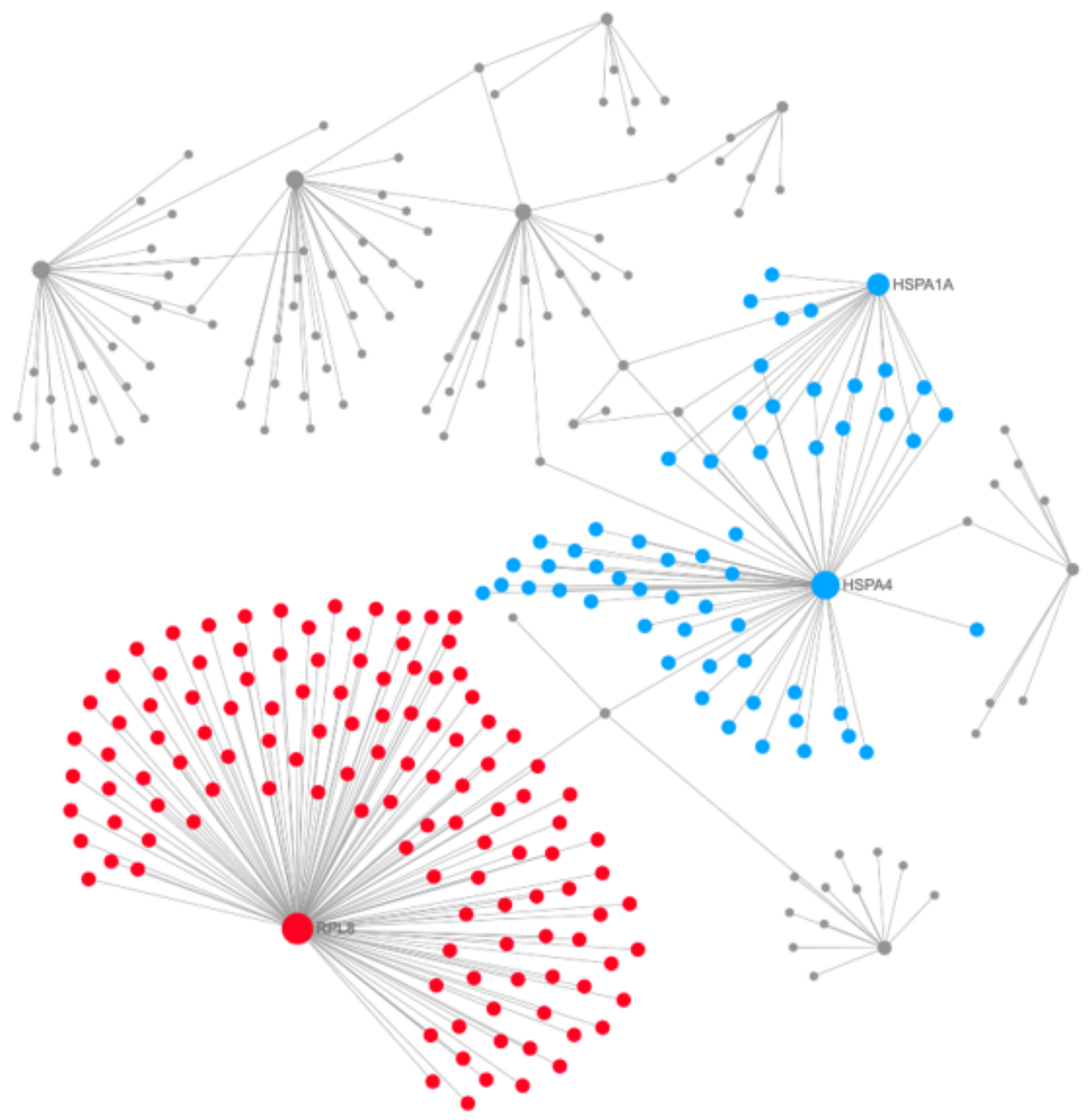

Figure 2

Modules 0 and 1 in the TCCSUPPi PPI network. The modules in blue and red are module 0 and module 1 respectively. The degrees of the nodes that connect to others in the network are represented by areas of the nodes. 

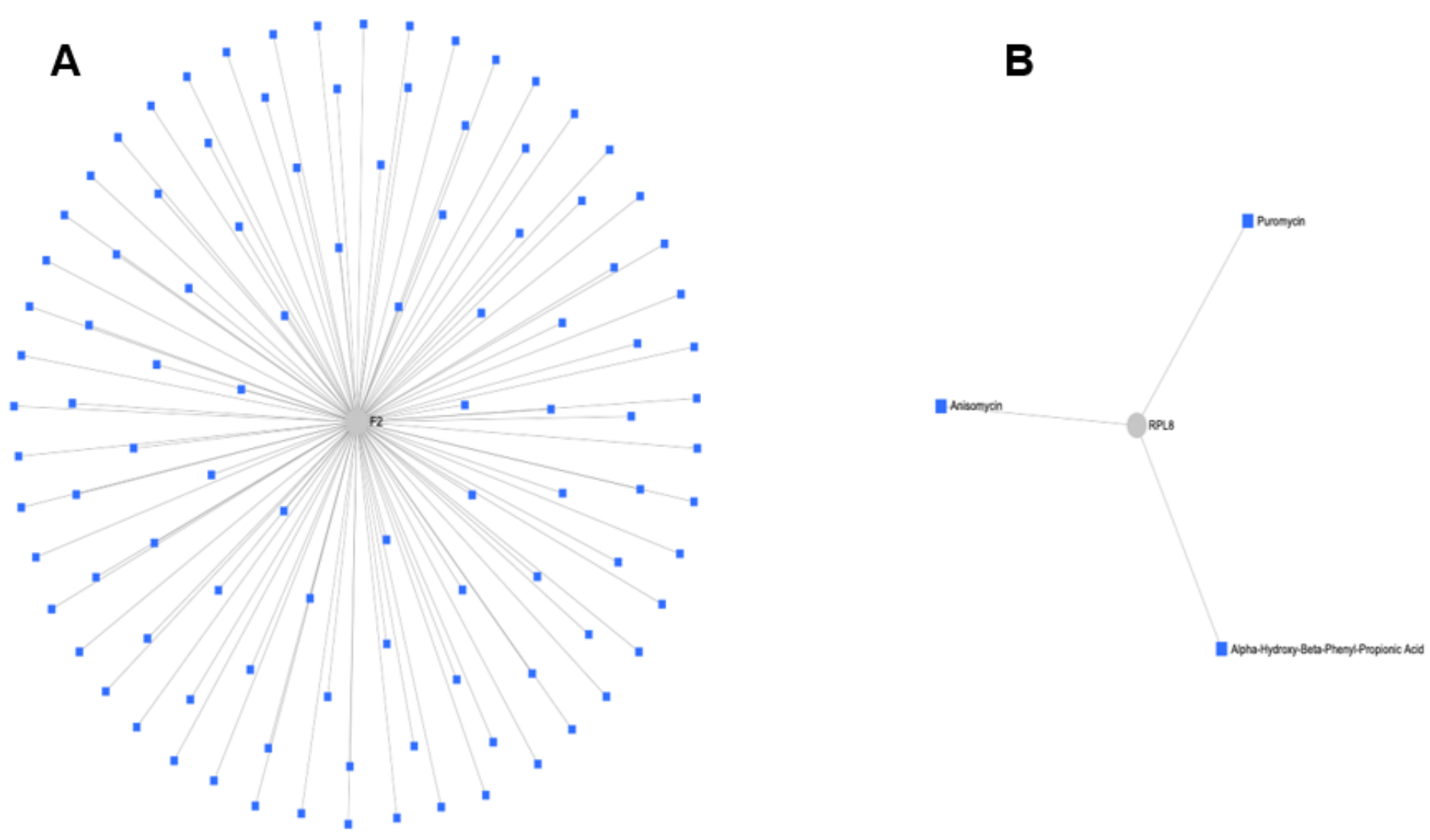

Figure 3

Protein-drug interaction network. A) The figure illustrates interactions between the downregulated node (F2) and several multiple drugs. B) The figure shows interactions between the upregulated node (RPL8) and three drugs 

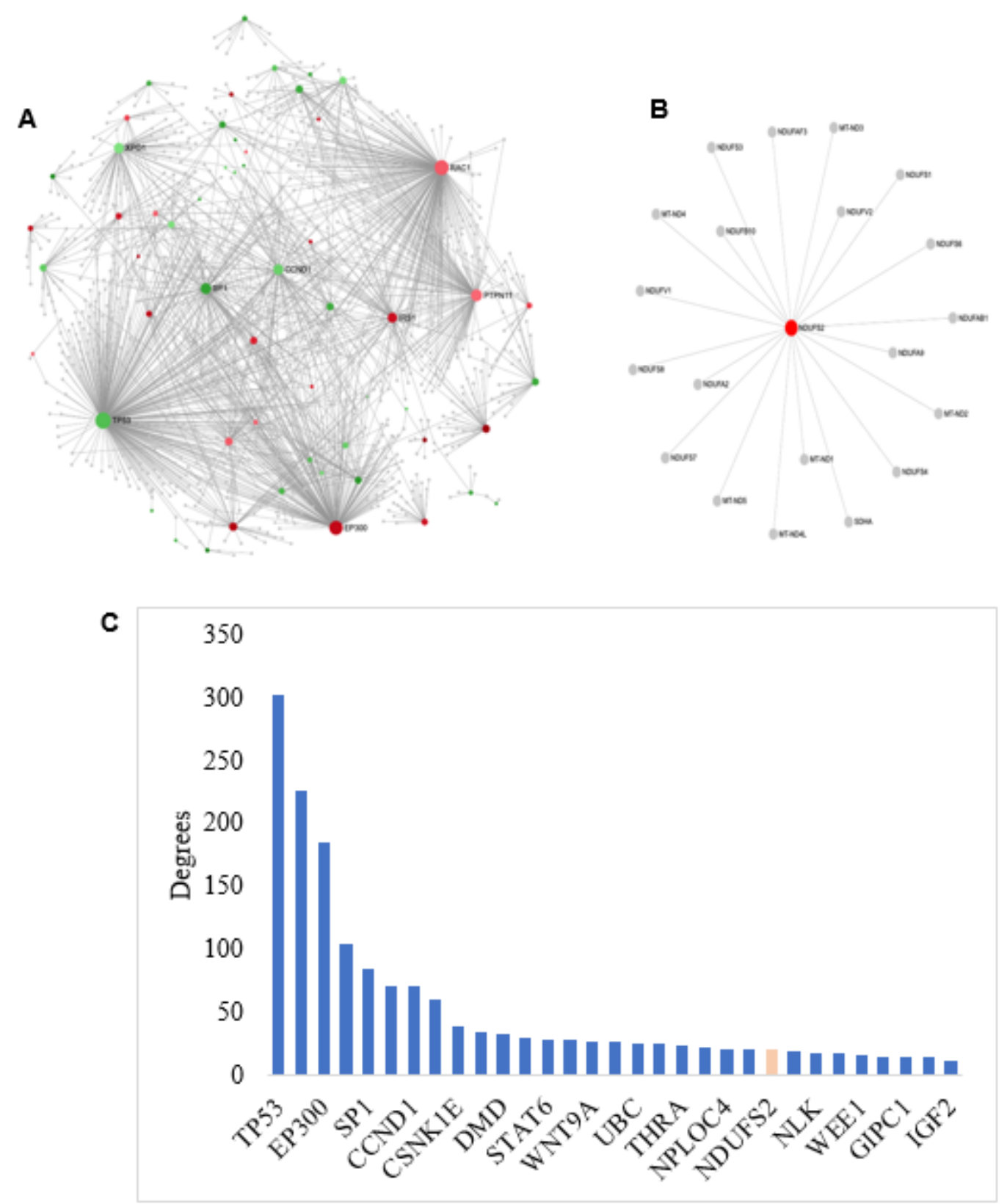

Figure 4

First and second identified networks in EJ28Pi cells. A) First identified network in EJ28Pi cells. Red and green colours represent the nodes expression that are up- and down-regulated. The expression level is represented by the colour grades and the area of the nodes indicate the degrees that the nodes connect to others. Nodes with gene names are the top 8 nodes in the PPI network. B) Second identified network in EJ28Pi cells. Subnetwork 2 showing only the upregulated node that is affected in the pathway. The red colour node is an indication that it is upregulated in EJ28Pi cells. C) Hub nodes in the PPI network in EJ28Pi cells. Top 16 hub nodes with their degree level are shown. Genes in blue colour are from subnetwork 1 and red accent are subnetwork 2. 


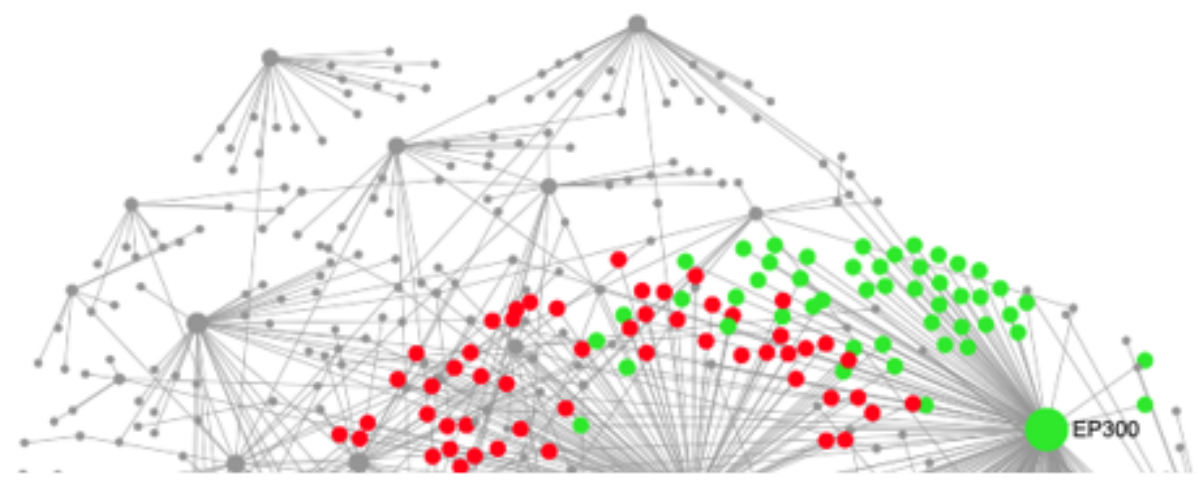

Figure 5

Modules 4, 5, 6, 7 and 8 in the EJ28Pi PPI network. The modules in red, blue, green, black and brown are for modules $4,5,6,7$, and 8 respectively. The degrees of the nodes that connect to others in the network is represented by areas of the nodes. 
A

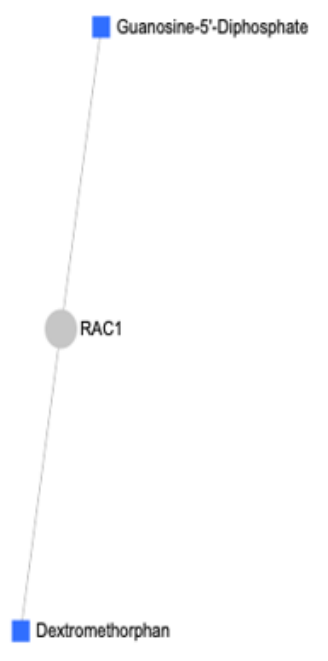

B

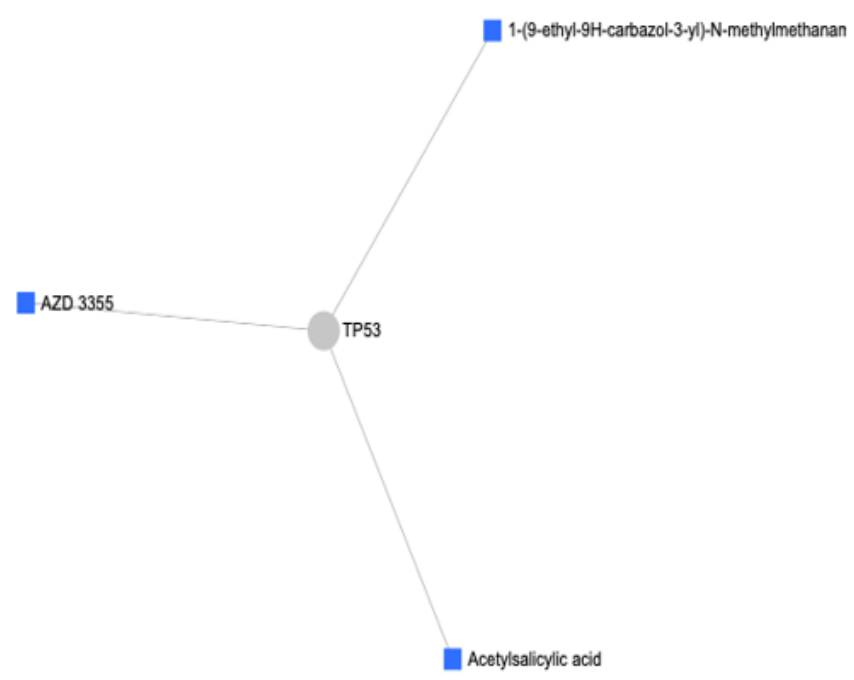

Figure 6

Protein-drug interaction network. A) The figure illustrates the interactions between upregulated node (RAC1) and two drugs. B) The figure shows interaction between upregulated node (TP53) and three drugs. 

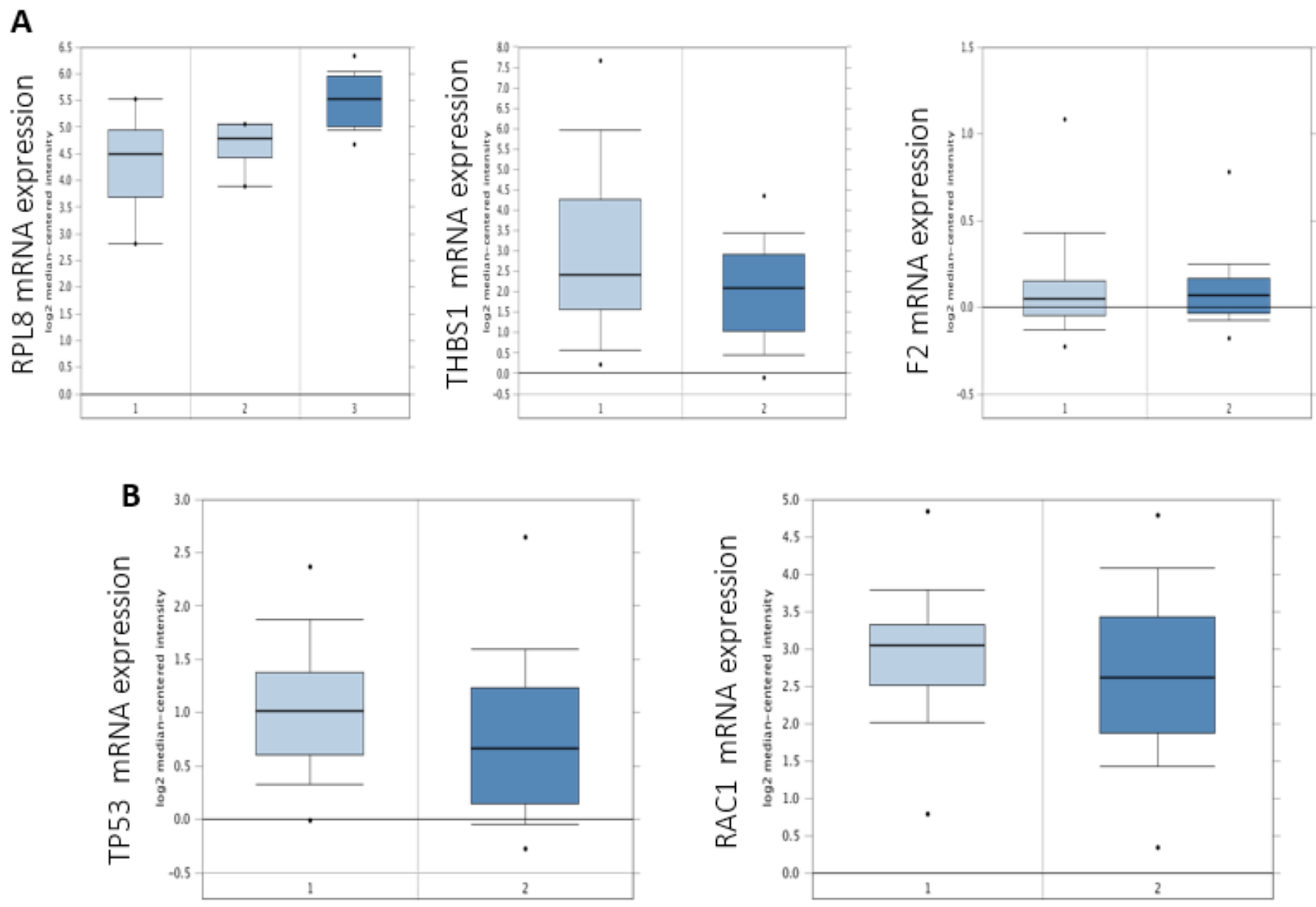

Figure 7

Expression of RPL8, THBS1, F2, TP53, and RAC1 mRNA levels obtained from TCCSUPPi and EJ28Pi. A) Expression of RPL8, THBS1 and F2 mRNA levels obtained from TCCSUPPi in bladder cancer using Oncomine. B) Expression of TP53 and RAC1 mRNA levels obtained from EJ28Pi in bladder cancer using Oncomine. Left plot is normal bladder tissue while the right plot is bladder cancer tissue.

\section{Supplementary Files}

This is a list of supplementary files associated with this preprint. Click to download.

- Supplimentarymaterials.docx 